\title{
Die Nachwirkung einer Bayvarolbehandlung auf später in die Bienenvölker eingebrachte Varroamilben
}

\author{
R Büchler *, V Maul \\ Hessische Landesanstalt für Tierzucht, Abteilung für Bienenzucht, Erlenstraße 9, \\ 3575 Kirchhain, Deutschland
}

(Eingegangen 11 Januar 1991; angenommen 10 Mai 1991)

\begin{abstract}
Zusammenfassung - Drei Versuchsgruppen von je 6 Völkern wurden über 3 Wochen mit je 8 Streifen Bayvarol behandelt. Nach 14, 28 bzw. 42 Tagen Wartezeit nach Ende der Bayvarolbehandlung wurden die Versuchsvölker einheitlich mit Milben infiziert. Als Kontrollgruppe dienten 6 Völker, die einige Wochen vor der Infektion nach der Bannwabenmethode behandelt worden waren. Der spontane Milbenabfall nach der Infektion und der Restbefall nach 21 Tagen wurden erfaßt. Der spontane Milbenabfall sinkt mit zunehmender Wartezeit und liegt in allen Versuchsgruppen deutlich höher als in der Kontrollgruppe. Der Zuwachs der Milbenpopulation zwischen Infektion und Restbefallsbestimmung steigt mit längerer Wartezeit an; für die Kontrollgruppe liegt er überraschend niedrig, geringfügig über dem der Versuchsgruppe mit 14-tägiger Wartezeit. Unterschiedliche Anteile heller Milben am Gesamtabfall bestätigen die zunehmende Abtötung der bei der infektion zugegebenen dunklen Milben bei verkürzter Wartezeit zwischen Bayvarolbehandlung und Infektion.
\end{abstract}

Apis mellifera / Varroabekämpfung / Bayvarol-Nachwirkung

\section{EINLEITUNG}

Der Wirkungsmechanismus des Bayvarol (a S: Flumethrin) beruht ebenso wie der des Apistan (a S: Fluvalinat) auf einer Verteilung des Wirkstoffs über die Körperoberfläche der Bienen, das Wabenwerk und den Beuteninnenraum durch die Aktivität der Bienen selbst. Die Milbe Varroa jacobsoni Oud (Acari, Varroidae) kommen durch den Kontakt zu den Bienen und den Wabenoberflächen in Berührung mit dem Wirkstoff (Koeniger, Fluchs, 1988). Moosbeckhofer und Kohlich (1990) haben für
Anwendungen von Apistan eine Nachwirkung der behandelten Bienen und des Wabenwerkes über die Behandlungsphase hinaus nachgewiesen. Moosbeckhofer (1991) stellt eine vergleichbare Nachwirkung Apistan- und Bayvarol-behandelter Waben kurz nach Behandlungsende fest.

In den bisher veröffentlichten Untersuchungen (Bogdanov et al, 1990; Moosbeckhofer, Kohlich, 1990) wurde vor allem die mögliche Bedeutung der Rückstände für eine Beschleunigung der Resistenzentwicklung bei der nachträglich wieder eindringenden Milbenpopulation und die

* Korrespondenz und Sonderdrucke 
Frage einer eventuellen Rückstandsbelastung des Honigs diskutiert. Die vorliegende Untersuchung zielt auf die Bestimmung der Dauer der Nachwirkung einer Bayvarolbehandlung. Diese ist von besonderem Interesse im Rahmen von Varroatoleranzprüfungen, bei denen nach Empfehlungen von Büchler (1989) Vergleichsvölker zu Prüfbeginn durch Behandlung (zum Beispiel mit Bayvarol) und nachfolgend gezielter Beimpfung mit ewa 100 Milben je Volk auf ein vergleichsbares Befallsniveau gebracht werden sollten.

\section{MATERIAL UND METHODEN}

Die Untersuchungen wurden mit normal entwickelten Völkern vergleichbarer Stärke in 3zargigen Holzmagazinbeuten durchgeführt. Zur Kontrolle des Milbenabfalls wurden ganzflächig abdeckende Bodeneinlagen verwandt. Die insgesamt 24 Völker eines separaten Standplatzes wurden zufällig in 4 Gruppen à 6 Volker aufgeteilt. Die Völker standen locker verteilt mit unterschiedlicher Ausflugsrichtung. Die Völker der Gruppen I-III wurden für 3 Wochen mit je 8 Streifen Bayvarol, verteilt über die 2 Brutzargen, behandelt. Die Völker der Gruppe I wurden im Zeitraum von 21 Mai bis 11 Juni behandelt, die der Gruppe II im Zeitraum von 04 Juni bis 25 Juni und die der Gruppe III im Zeitraum von 18 Juni bis zum 09 Juli 1990. Die Völker der Gruppe IV dienten als Kontrolle und wurden im Zeitraum vom 04 Juni bis zum 02 Juli nach dem Bannwabenverfahren (Maul et al, 1988) behandelt. Am 23 Juli, das heißt 6 Wochen nach Abschlu $B$ der Bayvarolbehandlung in Gruppe I, respektive 4 Wochen nach AbschluB der Bayvarolbehandlung in Gruppe II und 2 Wochen nach Abschluß der Bayvarolbehandlung in Gruppe III wurden alle Versuchsvölker einheitlich mit Milben gleicher Herkunft infiziert. Hierzu wurden die Bienen von 3 stark mit Varroa befallenen Völkern abgefegt und nach intensiver Durchmischung in gleichschwere Proben abgewogen. Jedem Versuchsvolk wurden 2 dieser Bienenproben zugegeben, 11 weitere dienten zur Bestimmung des Befallsgrades in den Proben. Dabei ergab sich ein mittlerer Milbenbefall von 215,4 je Probe mit einer Standardabweichung von $s=16,9$. Jedes Versuchsvolk hat danach im Mittel 431 Milben zugegeben bekommen. Alle Völker pflegten in der Zeit zwischen Startinfektion und Versuchsende Brut in allen Stadien.

Der nach der Infektion auftretende Milbentotenfall auf die Bodeneinlagen -im Folgenden als Spontanabfall bezeichnet- wurde im wöchentlichen Rhythmus erfaßt. Dabei wurden helle und dunkle, voll ausgefärbte Milben getrennt ausgezählt. Ab dem 14 August wurden alle Völker zur Bestimmung des Restmilbenbefalls für 3 Wochen mit Bayvarol behandelt. Der Milbentotenfall auf die Bodeneinlagen wurde wiederum im wöchentlichen Rhythmus und getrennt nach hellen und dunklen Milben bestimmt.

Die statistische Auswertung der Daten erfolgte mit dem Programmpaket SPSS (SPSS 1989). Abweichungen der behandelten Gruppen von der Kontrollgruppe wurden mittels $t$-Test geprüft.

\section{ERGEBNISSE}

In Tabelle I sind die Milbenabfallszahlen, getrennt nach dunklen und hellen Milben für den Zeitraum 14 Tage vor, 21 Tage nach der Startinfektion und während der Restbefallsbestimmung der verschiedenen Versuchsgruppen dargestellt.

Der geringe Milbenabfall vor der Startinfektion bestätigt die Effektivität der vorausgegangenen Behandlung in allen Versuchsgruppen. Eine nennenswerte, den Versuch möglicherweise störende Reinvasion kann nach dem Befund nicht festgestellt werden. Je kürzer die Wartezeit zwischen Bayvarolbehandlung und Infektion ist, um so höher liegt die Zahl spontan abgefallener Milben nach der Infektion. In allen bayvarolbehandelten Versuchsgruppen liegt der gesamte Spontanabfall deutlich höher als in der bannwabenbehandelten Kontrollgruppe. Die Gruppen I und III unterscheiden sich signifikant $(P<0.05)$ von der Kontrolle. Gruppe II liegt gerade oberhalb der Signifikanzschwelle, bedingt durch eine vergleichsweise hohe Varianz innerhalb der Gruppe. Aus unbekannter 


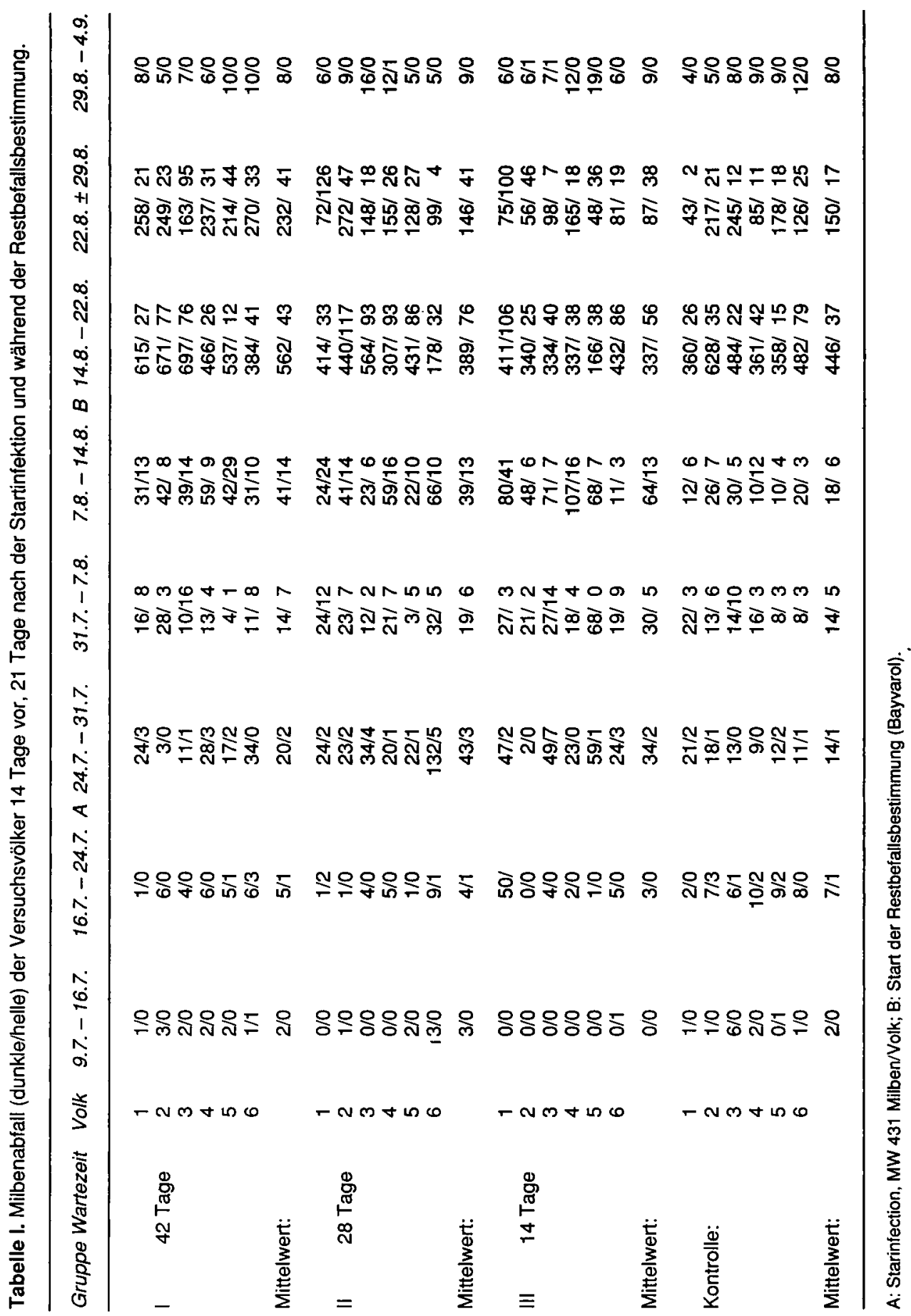


Ursache trat bei Volk 6 der Gruppe ein besonders hoher Spontanabfall auf. Der Restmilbenfall der bayvarolbehandelten Gruppen verhält sich erwartungsgemäß komplementär zu dem Spontanmilbenabfall, das heißt je mehr Milben spontan abgefallen sind, um so weniger Restmilben verbleiben im Volk. Die Korrelation zwischen Spontanabfall und Restmilbenbefall beträgt im Mittel der 3 bayvarolbehandelten Gruppen $r=-0,634$.

Im Gegensatz hierzu zeigt sich bei der bannwabenbehandelten Kontrollgruppe ein relativ niedriger Restmilbenbefall in Relation zu dem niedrigen Spontanmilbenabfall nach der Infektion. Die Korrelation zwischen Spontanabfall und Restmilben- befall für die Völker der Kontrollgruppe beträgt $r=0.398$.

In Abbildung 1 ist der mittlere Zuwachs der Milbenpopulation in den verschiedenen Versuchsgruppen angegeben, der sich ergibt, wenn man von dem Restbefall die Zahl der zugegebenen (im Mittel 431 Milben je Volk) und nicht spontan abgefallenen Milben subtrahiert (Restbefall-(Infektion-Spontanabfall)). Für die bayvarolbehandelten Gruppen liegt dieser Zuwachs erwartungsgemäß um so höher, je größer der zeitliche Abstand zwischen Infektion und vorheriger Bayvarolbehandlung ist. Bemerkenswerterweise ergibt sich für die Kontrollgruppe ein Zuwachs, der annähernd so niedrig liegt wie der der Völker

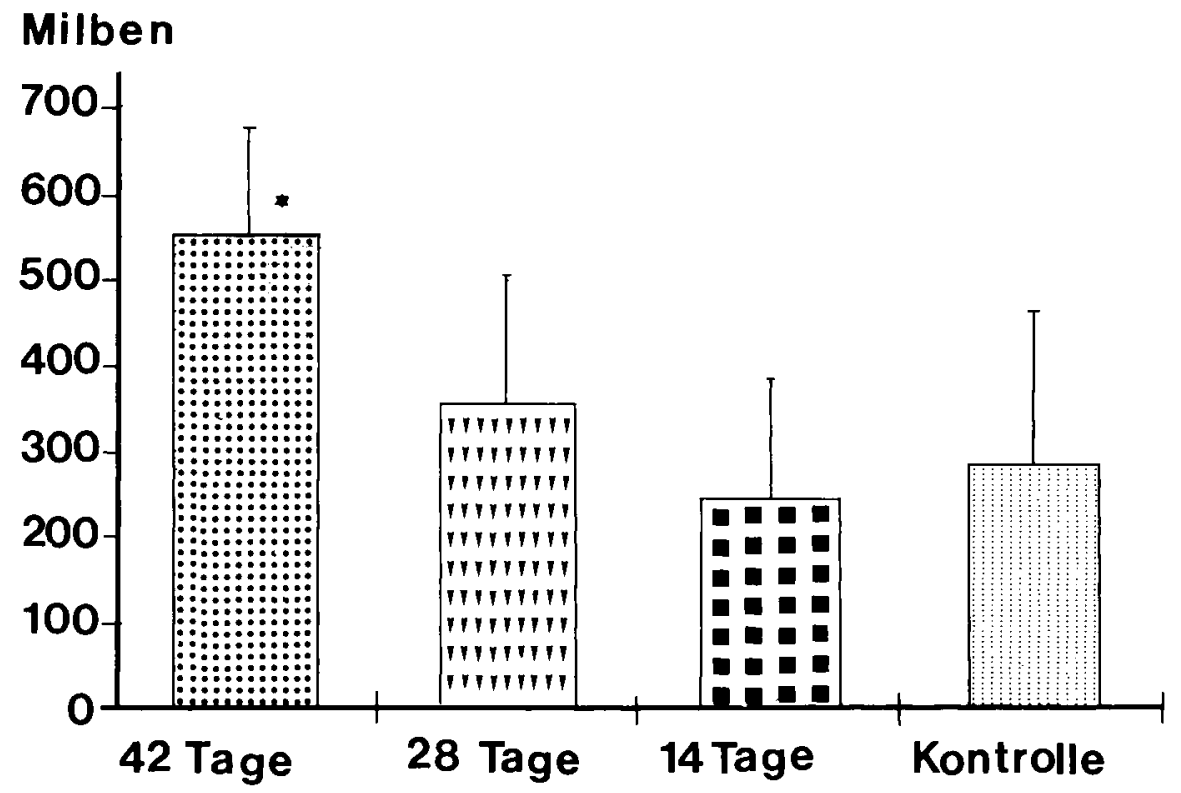

Wartezeit zwischen Bayvarolbehandlung und Infektion

Abb 1. Der Milbenzuwachs, ermittelt aus dem Restmilbenbefall nach Subtraktion der vorher zugegebenen ( $\bar{x} 431$ Milben/Volk) und nicht spontan abgefallenen Milben, in Versuchsgruppen mit unterschiedlicher Bayvarol-Rückstandsbelastung im Vergleich zu bannwabenbehandelten Kontrollvölkern. Anm: Standardabweichungen sind einseitig aufgetragen. " = Signifikante Abweichung von der Kontrolle ( $t$-Test, $P \leq 0,05)$. 
der Gruppe III mit nur 14 Tagen Wartezeit zwischen Bayvarolbehandlung und Infektion.

Die Auswertung der unterschiedlichen Milbenabfallszahlen wird sinnvoll ergänzt, wenn man die Anteile heller Milben am Spontanabfall und am Restmilbenbefall betrachtet (Abb 2). In den bayvarolbehandelten Gruppen ist der Anteil heller Milben am Spontanabfall umso niedriger, hingegen am Restbefall umso höher, je kürzer die Wartezeit zwischen Behandlung und Milbenzugabe ausfällt. Bei der Kontrollgruppe liegt der Anteil heller Milben sowohl beim Spontan- als auch beim Restbefall geringfügig unter dem der Gruppe mit der längsten Wartezeit.
Eine Auswertung des Milbentotenfalls im Verlauf der dreiwöchigen Bayvarolbehandlung zeigt, daß $71,2 \%$ der insgesamt abgetöteten Milben innerhalb der 1 Woche, $27,5 \%$ innerhalb der 2 Woche und nur $1,3 \%$ innerhalb der 3 Woche abfallen.

\section{DISKUSSION}

Die vorliegenden Ergebnisse bestätigen, daß nach Abschluß einer Bayvarolbehandlung eine Nachwirkung auf zugegebene Milben über Wochen festzustellen ist. Bei der hier geprüften Dosierung (8 Bayvarolstreifen, verteilt über 2 bienenbesetzte

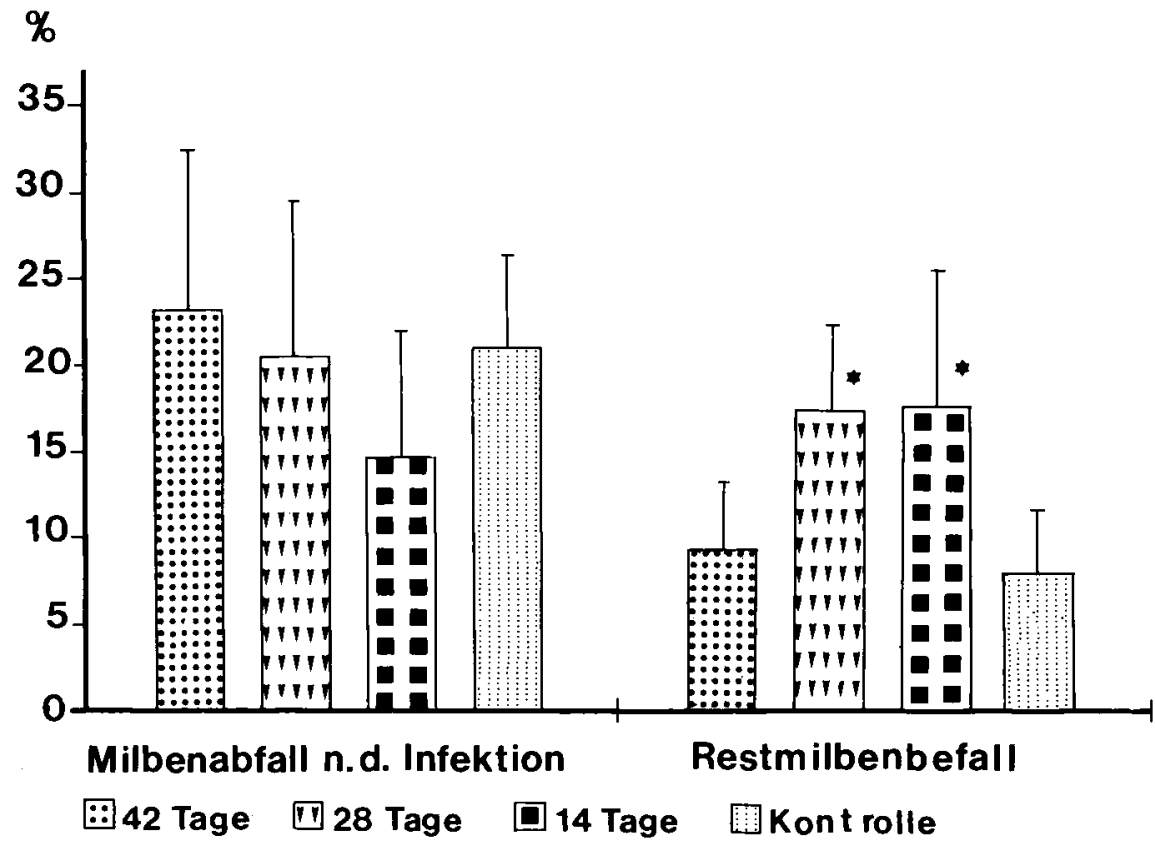

Wartezeit zwischen Bayvarolbehandlung und Infektion

Abb 2. Prozentualer Anteil heller Milben am spontanen Milbenfall innerhalb von 21 Tagen nach der Infektion und am Restmilbenbefall bei Völkern mit unterschiedlicher Bayvarol-Rückstandsbelastung im Vergleich zu bannwabenbehandelten Kontroilvölkern. Anm: Standardabweichungen sind einseitig aufgetragen. ${ }^{*}=$ Signifikante Abweichung von der Kontrolle $(t-$ Test, $P \leq 0,05)$. 
Brutzargen, 3 Wochen Anwendungsdauer) läßt sich erst nach etwa sechswöchiger Wartezeit ein weitgehendes Nachlassen der Wirkung auf wieder zugegebene Milben beobachten. Diese Frist sollte zwischen einer Behandlung der Völker mit Bayvarol und einer gezielten Neuinfektion, wie sie zum Vergleich der Varroatoleranz von Prüfvölkern im Rahmen von Selektionsvorhaben zu empfehlen ist (Büchler, 1989) mindestens eingehalten werden. Moosbeckhofer (1991) befürchtet aufgrund seiner Untersuchung beim Apistan sogar erheblich längere Fristen.

Um die Nachwirkintensität zu minimieren, sollte die Behandlungsdauer möglichst kurz gehalten werden. Der Verlauf der Milbenabtötung in dem vorliegenden Versuch zeigt, daß ein auf 14 Tage befristeter Einsatz der Streifen unter bestimmten Umständen zum Erreichen einer hohen Wirksamkeit völlig ausreichen kann.

Die vergleichsweise niedrige Zuwachsrate der zugegebenen Milbenpopulation in den bannwabenbehandelten Kontrollvölkern stellt eine interessante Nebenbeobachtung des Versuches dar. Die bannwabenbehandelten Völker pflegten zum Zeitpunkt der Milbenzugabe genau wie die bayvarolbehandelten Vergleichsgruppen Brut in allen Stadien. Unterschiede der Völker in ihrer Funktion als Wirtstier für Varroa sind daher eher im Zusammenhang mit der veränderten Altersstruktur der Bienen in den bannwabenbehandelten Völkern zu sehen. Deren Auswirkungen auf die Verteilung der Milben zwischen adulten Bienen und Brut, die Reproduktionsneigung der Milben und den Milbenabgang durch Flugbienenverlust sind in Betracht zu ziehen und soliten durch weitere Untersuchungen geklärt werden.

Unabhängig von der korrekten Erklärung des Phänomens kann als Auswirkung von Brutpausen im Sommer auf die Varroa-population generell angenommen werden, daß es neben dem befristeten Entzug der Reproduktionsbasis zu Veränderungen im Bienenvolk kommt, die die Entwicklung der Milbenpopulation beeinträchtigen.

\section{Summary - The after-effect of Bayvarol treatment in honey bee colonies on Var-} roa mites introduced later on. The system of Varroa control by means of Bayvarol and Apistan is based on a constant distribution of active agent within the whole colony during the treatment period. Moosbeckhofer and Kohlich (1990) and Moosbeckhofer (1991) have first shown that residues with toxic effects on mites remain on bees and on combs after treatments with Apistan and Bayvarol. Such aftereffects also have to be considered in experiments using systematic inoculation of test colonies with Varroa mites in order to determine differences in Varroa tolerance of bees. The present study aims at the determination of the necessary waiting period between Bayvarol treatment and a new experimental inoculation.

Within one apiary, 3 groups of 6 colonies each were subsequently treated over 3 wk with 8 strips of Bayvarol (1: 21 May11 June; II: 4 June-25 June; III: 18 June-9 July). In a fourth group (control) mites were removed by means of the trapping-combtechnique (Maul et al, 1988).

On 23 July, all colonies were inoculated with weighed samples of infested bees, containing an average of 431 Varroa mites each. Mite mortality was determined weekly by means of protected inserts. Light and dark mites were counted separately. After another Bayvarol treatment from 14.08.04.09., the total of residual mites was determined for all colonies.

The results show clearly a dependance of the spontaneous mite mortality from the lengths of the waiting period (numbers of dark/light mites in table I). Group I with the 
longest waiting period has the lowest spontaneous mite mortality and the highest number of residual mites 3 wk after inoculation. The numerical increase in the Varroa population [residual mites - (inoculated mites - spontaneously killed mites)] (fig 1) is correlated with the waiting period and is surprisingly low in the control group compared to group I. This indicates an influence of the trapping comb treatment on the subsequent Varroa reproduction, which so far has not been recorded.

Finally, the percentage of light mites (immature daughters), an indicator of Varroa reproduction, is shown in figure 2 . The percentage of light mites from the spontaneously killed mites is higher and from the residual mites is lower for groups with a longer waiting period.

It is concluded from the results that after Bayvarol treatment under the tested conditions a 6-wk-waiting period is necessary before new mites can be safely inoculated. in this experiment $98.7 \%$ of the total treatment effect was achieved within $14 \mathrm{~d}$.

The phenomenon of a reduced Varroa reproduction after the application of trapping-comb treatment is assumed to be connected with the different age structure of the bee population in this group.

\section{Apis mellifera / Varroa jacobsoni /} chemical control / after-effect / Bayvarol

Résumé - Effet ultérieur d'un traitement au Bayvarol de colonies d'abeilles sur les acariens Varroa introduits par la suite. La lutte contre Varroa jacobsoni (Acari, Varroidae) à l'aide du Bayvarol et de l'Apistan est basée sur la distribution constante de la substance active à l'intérieur de toute la colonie durant la période de traitement. Moosbeckhofer et Kohlich (1990) et Moosbeckhofer (1991) ont montré les premiers que des résidus toxiques pour les acariens étaient présents sur les abeilles et les rayons après les traitements à l'Apistan et au Bayvarol. De tels effets ultérieurs doivent être pris en compte dans les expériences où l'on infeste systématiquement des colonies tests avec des varroas pour déterminer les différences de sensibilité des abeilles à cet acarien. Notre étude cherche à déterminer la période de latence nécessaire entre un traitement au Bayvarol et une nouvelle infestation expérimentale. Au sein d'un même rucher, 3 groupes de 6 colonies ont été traités pendant 3 semaines avec 8 rubans de Bayvarol (I : du 2105 au 11 06; II : du 0406 au 25 06; III : du 1806 au 09 07). Dans le $4^{e}$ groupe (témoin) les acariens ont été éliminés par la méthode du rayon-piège (Maul et al, 1988). Le 23 juillet, toutes les colonies ont reçu une même quantité d'abeilles infestées en moyenne par 431 varroas. Un nouveau traitement au Bayvarol du 1408 au 0409 a permis de déterminer le nombre d'acariens résiduels dans chaque colonie. Les résultats montrent clairement que la mortalité spontanée des acariens dépend de la durée de la période de latence (nombre d'acariens foncés/clairs du tableau I). Le groupe I, qui avait eu la plus longue période de latence, a eu la plus faible mortalité spontanée de varroas et le plus grand nombre de varroas résiduels 3 semaines après l'infestation. L'accroissement numérique de la population de varroas [varroas résiduels - (varroas introduits - varroas morts spontanément)] est corrélé avec la période de latence et particulièrement faible chez le groupe témoin par rapport au groupe I. Cela indique une influence de la méthode du rayon-piège sur la reproduction du varroa, qui jusqu'à présent n'a pas été mentionnée. La figure 2 donne le pourcentage d'acariens clairs (filles immatures), qui est un indicateur de la reproduction de Varroa. Plus la période de latence est longue et plus le pourcentage d'acariens clairs est élevé chez les acariens morts spontanément, et faible 
chez les acariens résiduels. On en conclut que, dans les conditions expérimentales présentes, une période de 6 semaines est nécessaire après un traitement au Bayvarol avant que de nouveaux acariens puissent être introduits en toute sécurité. Dans l'expérience présente, $98,7 \%$ des acariens tués l'ont été pendant les 2 premières semaines du traitement. On pense que la réduction de la reproduction de Varroa après utilisation de la méthode du rayon-piège est liée à la structure de la population d'abeilles en fonction de l'âge.

Apis mellifera / Varroa jacobsoni / lutte chimique / effet ultérieur / Bayvarol

\section{LITERATUR}

Bogdanov S, Imdorf A, Kilchenmann V, Gerig L (1990) Rückstände von Fluvalinat in Bienen- wachs, Futter und Honig. Bienenwelt 32 (5), 110-113

Büchler R (1989) Die Auslese und Zucht varroaresistenter Bienen-Bericht aus dem laufenden Forschungsprojekt, Vortrag anläßlich der Züchtertagung des DIB am 12 März 1989 in Saarbrücken. Der Imkerfreund 44, 197-201

Koeniger N, Fuchs S (1988) Control of Varroa jacobsoni Oud in honeybee colonies containing sealed brood cells. Apidologie 19, 117-130

Maul V, Klepsch A, Assmann-Werthmüller U (1988) Das Bannwabenverfahren als Element imkerlicher Betriebsweise bei starkem Befall mit Varroa jacobsoni Oud. Apidologie 19, 139-154

Moosbeckhofer R, Kohlich A (1990) Nachwirkung von Apistan nach der Entfernung der Streifen! Bienenvater 111, 3-9

Moosbeckhofer R (1991) Apistan und BayvarolLangzeitwirkung behandelter Waben. Die Biene, 127 (6), 331-334

SPSS (1989) SPSS/PC + Version 3.0. SPSS $\mathrm{GmbH}$, Steindorfstraße 19, 8000 München 22 